\title{
Cold atmospheric plasma coupled with air abrasion in liquid medium for the treatment of peri-implantitis model grown with a complex human biofilm: an in vitro study
}

\author{
Wang Lai Hui ${ }^{1,2} \cdot$ Vittoria Perrotti $^{3}\left(\mathbb{D} \cdot\right.$ Adriano Piattelli $^{3} \cdot$ Kostya (Ken) Ostrikov $^{4} \cdot$ Zhi Fang $^{5} \cdot$ Alessandro Quaranta $^{1,2,6}$
}

Received: 15 February 2021 / Accepted: 13 April 2021 / Published online: 24 April 2021

(C) The Author(s) 2021

\begin{abstract} preservation of surface topography. multiple comparison test. alterations on the implant surface in all the groups.

Vittoria Perrotti

v.perrotti@unich.it

1 Smile Specialists Suite, Newcastle, NSW, Australia

2 Formerly, School of Dentistry and Oral Health, Griffith University, Gold Coast, Queensland, Australia

3 Department of Medical, Oral and Biotechnological Sciences (DSMOB), University of Chieti-Pescara, Via dei vestini, 31, 66100 Chieti, Italy

4 School of Chemistry and Physics, Queensland University of Technology, Brisbane, Queensland 4000, Australia

5 College of Electrical Engineering and Control Science, Nanjing Tech University, Nanjing 210009, China

6 Sydney Dental Hospital, Sydney, NSW, Australia
\end{abstract}

Objective Treatment of implants with peri-implantitis is often unsuccessful due to residual microbial biofilm hindering reosseointegration. The aim of this study was to treat biofilm-grown titanium (Ti) implants with different modalities involving air abrasion (AA) and cold atmospheric plasma (CAP) to compare the effectiveness in surface decontamination and the alteration/

Materials and methods Saliva collected from a peri-implantitis patient was used to in vitro develop human biofilm over 35 implants with moderately rough surface. The implants were then mounted onto standardized acrylic blocks simulating periimplantitis defects and treated with AA (erythritol powder), CAP in a liquid medium, or a combination (COM) of both modalities. The remaining biofilm was measured by crystal violet (CV). Surface features and roughness before and after treatment were assessed by scanning electron microscope (SEM). The data were statistically analyzed using Kruskal-Wallis followed by Tukey's

Results In the present peri-implantitis model, the human complex biofilm growth was successful as indicated by the statistical significance between the negative and positive controls. All the treatment groups resulted in a remarkable implant surface decontamination, with values very close to the negative control for AA and COM. Indeed, statistically significant differences in the comparison between the positive control vs. all the treatment groups were found. SEM analysis showed no post-treatment

Conclusions Decontamination with AA delivering erythritol with or without CAP in liquid medium demonstrated compelling efficacy in the removal of biofilm from implants. All the tested treatments did not cause qualitative alterations to the Ti surface features. No specific effects of the CAP were observed, although further studies are necessary to assess its potential as monotherapy with different settings or in combination with other decontamination procedures.

Clinical relevance CAP is a promising option in the treatment of peri-implantitis because it has potential to improve the elimination of bacterial plaque from implant surfaces, in inaccessible pockets or during open-flap debridement, and should stimulate

the process of the re-osseointegration of affected dental implants by not altering surface features and roughness.

Keywords Air abrasion · Biofilm · Decontamination · Dental implants · Plasma

\section{Introduction}

Biofilm-induced implant disease, peri-implantitis, has been reported to occur in $1-47 \%$ of all implants placed globally [1]. Despite the high prevalence, currently scientists are still in perusal for a universally accepted and reliable treatment modality. Peri-implantitis is an inflammatory reaction of the host to bacteria and presents 
clinically with pathological bone loss around dental implants which often results in saucer-shaped bony defects [2]. The ultimate outcome of untreated or failed treatment of peri-implantitis is the loss of the implant. While regular professional and home care can maintain healthy peri-implant tissues, once a mature biofilm has established itself on the subgingival implant surface, it becomes a big challenge for management [3]. This is because the implant surface is both macroscopically and microscopically rough, which makes it notoriously difficult to clean. Indeed, early dental implants used to have a simple threaded screw forms and plain, machined surfaces which were unmodified after milling, while almost all modern dental implants are designed to have rough surfaces with features that increase the surface area and energy. The rationale is to enhance the adhesion of blood, matrix proteins, and human cells [4]. Altering the surface of a titanium (Ti) implant to increase its roughness does not compromise its biocompatibility but enhances the total area available for host cells attachment, hence integration with bone osseointegration [5]. Unfortunately, these altered implant surfaces with complex topographies do not differentiate between prokaryotes and eukaryotes. In other words, both bacterial and host cells can attach to the implants simultaneously. This effect promotes biofilm formation, the leading etiology of peri-implantitis.

Despite many trials on peri-implantitis treatments, multiple systematic reviews have yielded the same conclusion that based on the current evidence, no particular treatment can be established as a gold standard approach for the treatment of peri-implantitis [6-9]. Since the treatment of peri-implantitis aims to achieve both decontamination and reosseointegration, it is necessary to remove not only all viable bacteria but all traces of bacterial products such as endotoxins, in order to maximize the likelihood of success. Traditional periodontal treatments using scaling instruments to remove biofilms originally designed for debriding the roots of natural teeth cannot be applied in the same manner to threaded implant surfaces. The implant surface is far more protected and inaccessible to conventional professional instruments [10]; this lack of cleaning efficacy is worse in non-surgical approach. This is particularly true when the peri-implant lesions are compounded by unfavorable defect configurations, for example, the typical deep and narrow bony defects; these findings necessitated exploration of surgical options [11]. As shown in various systematic reviews $[12,13]$, while nonsurgical treatment has been shown to be effective in resolving peri-implant mucositis, it does not reliably resolve periimplantitis. Peri-implantitis is best managed surgically.

Nevertheless, whether it is conservative, resective, or regenerative treatments, surgical approaches would only be successful if they are executed in conjunction with effective implant surface decontamination [11]. Access to the implant surface may be improved by surgical means, but it is still far from ideal; most of the techniques proposed in the scientific literature are limited by lack of adequate access. In vitro peri-implantitis models stained with ink and tested for air abrasion (AA) with several powder particles reported to have the superior surfaces of the implant threads more readily cleaned, whereas the inferior surfaces are often observed to retain residual dye $[10,14]$. Dental laser applications in treating periimplantitis are also exploring side-firing tips to more efficiently access the under-surface of the implant threads $[15,16]$. AA has demonstrated its superior biofilm decontaminating power on flat titanium discs, but its efficacy in peri-implantitis model is yet to be tested [17].

Cold atmospheric plasma (CAP) is an innovative, yet earlystage, treatment modality in the oral implantology field. In a recent review [18] based on 23 studies investigating the effects of CAP on biocompatibility, surface improvement, and cleaning efficacy of implant surfaces, it was suggested that CAP is a promising option for the treatment of periimplantitis [19, 20], although further evidence is necessary to draw final conclusions. The hypothesized benefits in incorporating CAP for peri-implantitis treatment is that it can act via a liquid medium. The liquid environment, in which the cells are normally located, acts as an interface between plasma and living matter. CAP can activate a liquid interface with reactive species which then act as a carrier agent to deliver the antibacterial effects onto the underlying target surface, as simulated by dental implants contaminated by biofilm treatment in a combination of saliva, gingival crevicular fluid, and even blood. Tresp et al. (2013) [21] showed superoxide anion radicals $\left(\mathrm{O}_{2}{ }^{\circ}\right)$, as well as hydroxyl radicals $\left({ }^{\circ} \mathrm{OH}\right)$ were generated in plasma-treated phosphate-buffered saline solution. Earlier in 2010, Liu et al. [22] successfully inactivated Staphylococcus aureus suspended in a liquid using a directcurrent CAP microjet. This finding has prompted the potential use of CAP in treating peri-implantitis despite the lack of direct contact between the plasma jet and the implant surface.

The aim of this study was to treat biofilm-grown $\mathrm{Ti}$ implants in a peri-implantitis model with different modalities involving AA and CAP in order to compare their efficacy in surface decontamination and the effects of those decontamination on Ti surface features.

\section{Materials and methods}

\section{Titanium implants}

A total of 35 grade 4 pure Ti dental implants $(4.1 \times 10 \mathrm{~mm})$ with a moderately rough surface blasted with alumina 
particles (Southern Implants ${ }^{\circledR}$, Irene, South Africa) were adopted for the present study.

\section{Study design}

The protocol of the present study was approved by the Griffith University Human Research Ethics Committee (GU Ref No: 2018/35).

The 35 Ti implants are subdivided into 5 treatment groups (Fig. 1):

- $\quad$-ve control group: non-contaminated and treated by AA and CAP (\#2)

- $\quad$ +ve control group: contaminated and untreated (\# 3)

- AA: contaminated and treated the by AA (\#10)

- CAP: contaminated and treated by CAP (\#10)

- COM: contaminated and treated by the combined AA and CAP treatment (\#10)

\section{Biofilm growth}

A systemic healthy volunteer with no evidence of active caries, no salivary gland disease, but affected by periimplantitis was selected to donate his saliva. The subject was asked to refrain from practicing oral hygiene routine for $12 \mathrm{~h}$ before saliva collection in the morning. The subject chewed on paraffin wax until $5 \mathrm{~mL}$ of saliva were collected.

To generate biofilm, Ti implants were incubated in $1 \mathrm{~mL}$ of Brain Heart infusion (BHI) media (OXOID CM1135, 37gr/L) supplemented with 5\% defibrinated sheep blood [23] and 10\% human saliva from the donor $[24,25]$ in sterile 24-well plates (Costa 3524) for $96 \mathrm{~h}$ under anaerobic conditions at $37^{\circ} \mathrm{C}, 80 \mathrm{rpm}$ to allow bacterial growth. For negative controls, implants were incubated at the same conditions with $1 \mathrm{~mL}$ of BHI supplemented with 5\% defibrinated sheep blood and $10 \%$ PBS solution, but without human saliva.

\section{Peri-implantitis model}

Prior to biofilm growth, the apical $4 \mathrm{~mm}$ of the $35 \mathrm{im}$ plants was coated with parafilm wax (Fig. 2a). As the biofilm matured on the implant surface, the parafilm wax was removed; hence, only the coronal $6 \mathrm{~mm}$ of the implant surface and the tissue level machined collar were contaminated with biofilm growth. Each implant was mounted in an acrylic resin block (Sawbones, Vashon Island, WA, USA) prepared with 6-mm-deep defects with a circumferential saucer-shaped opening at $60^{\circ} \mathrm{C}$ (Fig. 2b) to simulate the physical environment of a peri-implantitis lesion [14]. These defects were in the same morphology as the Class Ie defects described by Schwarz et al. [26]. When the implants were inserted into the pre-fabricated defects, three full threads were exposed in the coronal region.

\section{Instrumentation modalities}

\section{Air abrasion}

Implants were treated on 4 surfaces with $90^{\circ}$ rotation per surface in accordance with the acrylic resin block. An AA device (Airflow ${ }^{\circledR}$ Prophylaxis Master, EMS, Nyon, Switzerland) at a static pressure 7 bar (101.5 psi) using erythritol powder (particle size $14 \mu \mathrm{m}$ ) was used to treat the implants for $20 \mathrm{~s}$ in each surface, $10 \mathrm{~s}$ upwards and $10 \mathrm{~s}$ downwards motion with the nozzle head angulated towards the mid-point of the treated implant surface, at a distance of $10 \mathrm{~mm}$ and $60 \mathrm{~mL}$ water $/ \mathrm{min}$. A standard handpiece (Airflow ${ }^{\circledR}$, EL-308/C, EMS Nyon, Switzerland) was mounted with a holder so as to keep

\begin{tabular}{|c|c|c|c|c|c|}
\hline CV & -ve control & +ve control & AA & CAP & COM \\
\hline 1 & O & O & 0 & O & 0 \\
\hline 2 & 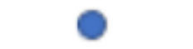 & ○ & O & 0 & 0 \\
\hline 3 & & & O & 0 & - \\
\hline 4 & & & ○ & 0 & - \\
\hline 5 & & & 0 & 0 & ○ \\
\hline 6 & & & ○ & 0 & ○ \\
\hline 7 & & & ○ & - & - \\
\hline 8 & & & O & 0 & 0 \\
\hline SEM & -ve control & +ve control & AA & CAP & COM \\
\hline 1 & & 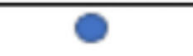 & O & $\overline{0}$ & $\overline{-1}$ \\
\hline 2 & & & O & 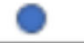 & 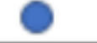 \\
\hline
\end{tabular}

Fig. 1 Schematic drawing of the study design 
Fig. 2 a Parafilm wax coating of implant's apical $4 \mathrm{~mm}$. b Periimplantitis model of Sawbone defect
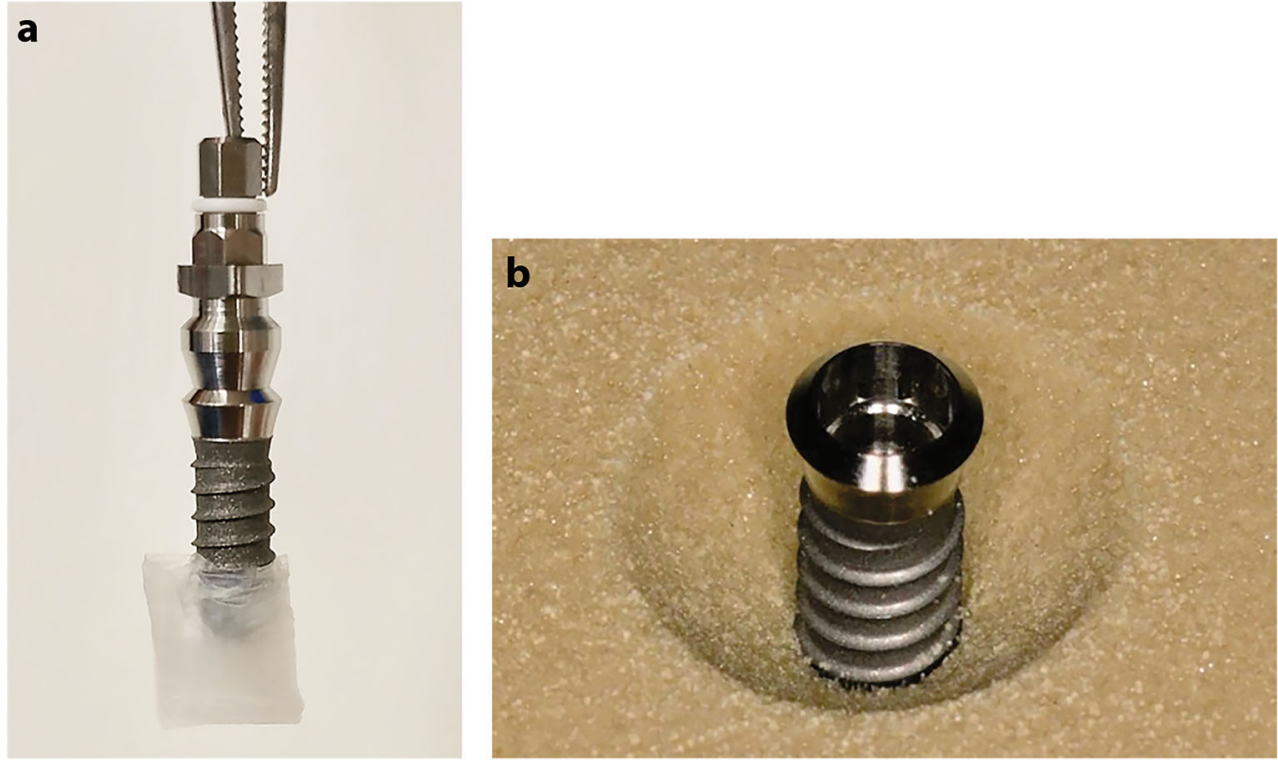

the nozzle at a static position to each Ti implants treated. The holder allows rotation of the handpiece along its own axis so as to ensure that ejected water, powder, and air are projected along the implant surface (Fig. 3a-b). All treatments were performed by the same experienced operator, a senior periodontics specialist in training (W.H.L.).

\section{Cold atmospheric plasma}

Plasma treatment was performed with an experimental spark plasma pen jet previously described in detail in Hui et al. [27]. The pen jet was mounted on a standardized holder keeping a distance of $5 \mathrm{~mm}$ from the plasma nozzle tip (end of discharge capillary) to the $\mathrm{Ti}$ implants surface. The plasma pulses were delivered with the repetition frequency of $1.4 \mathrm{~Hz}$. A high RF voltage $(10 \mathrm{kV})$ was coupled to the needle electrode. The temperature was maintained very close to the room temperature (RT), with the deviation not exceeding $2{ }^{\circ} \mathrm{C}$ at the tip of the plasma jet. All roughened Ti implants surfaces were submerged in PBS solution to ensure that the liquid medium smoothly covers all the imperfections (e.g., rough surface features) on the sample surface (Fig. $4 a-c)$.

\section{Biofilm quantification}

Eight implants belonging to groups AA, CAP, and COM and 2 implants for negative and positive controls were assessed by crystal violet (CV) assay [28] to evaluate the effect of different treatment modalities on biofilm removal; experiments were repeated in triplicates. Implants were first washed twice with $500 \mu \mathrm{L}$ of sterile distilled water. The biofilm was then stained with $500 \mu \mathrm{L}$ of $0.1 \%$ $\mathrm{CV}$ for $30 \mathrm{~min}$ at room temperature in a 24-well plate. The implants were than washed three times with $1 \mathrm{~mL}$ of sterile distilled water and removed maximum water possible by tapping onto paper towel. The dye bound to adherent biofilm was then solubilized using $500 \mu \mathrm{L}$ of ethanol to acetone ratio of $80 \%: 20 \%$, and the optical density
Fig. 3 a Schematic drawing of air abrasion treatment. b Setup of PI model and air abrasion treatment
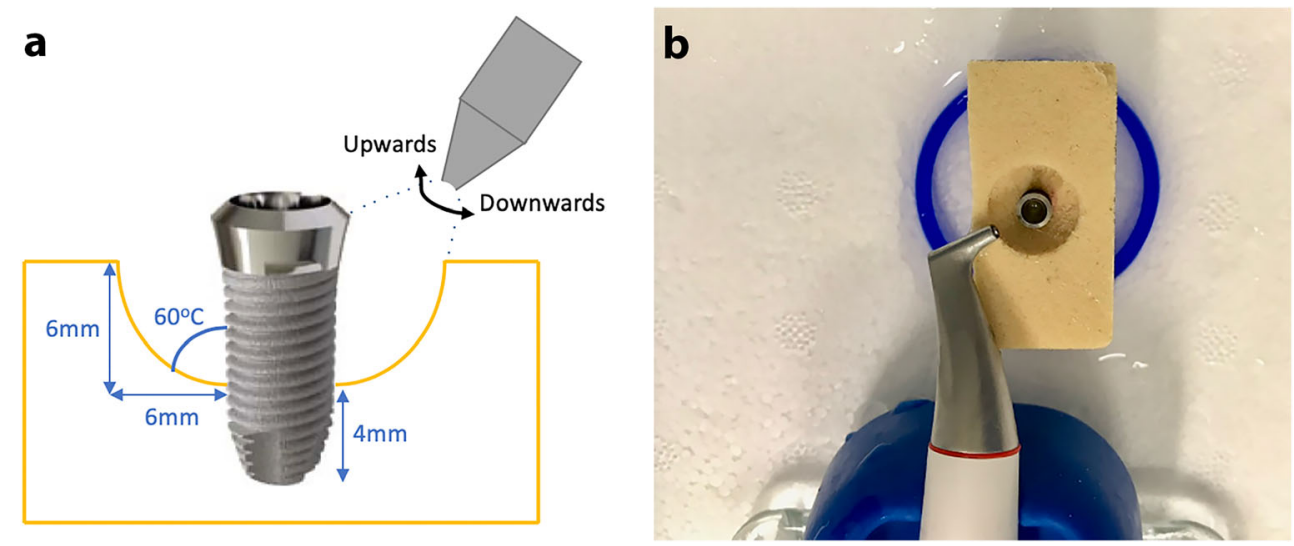
(OD) of the solubilized dye was measured at $595 \mathrm{~nm}$ with a plate reader (POLARstar Omega plate reader, BMG Labtech, Adelaide, Australia). Ethanol to acetone ratio was used as the blank to subtract any background reading. The percentage reduction in biofilm caused by the treatment was calculated as follows:

$\left(\left(\mathrm{OD}_{+\mathrm{ve} \text { control }}-\mathrm{OD}_{\text {treatment }}\right) / \mathrm{OD}_{+\mathrm{ve} \text { control }}\right) \times 100 \%$.

\section{Measurement of surface topography}

Pristine implants (uncontaminated and untreated) were evaluated by scanning electron microscope (SEM, Thermal Field Emission SEM, JEOL JSM-7100, Tokyo, Japan) to provide a baseline surface assessment and description prior to group allocation. The samples were rinsed with $0.1 \mathrm{M}$ of phosphate-buffered solution (PBS) at $\mathrm{pH} 7.1$ and fixed overnight with a $4 \%$ PBSparaformaldehyde solution at $4{ }^{\circ} \mathrm{C}$. Samples were further washed with PBS buffer and dehydrated using an ascending alcohol series before mounting onto aluminum stubs and gold sputtering in an Emitech K550 (Emitech Ltd., Ashford, Kent, UK).

A SEM with $10 \mathrm{kV}$ and $3.3 \mathrm{~A}$ was used to capture images of the implants surface before and after the treatments. These images were used to examine any alteration on the Ti surface and were captured at 3, 6, 9, and 12 o'clock positions, at a distance of $300 \mathrm{~mm}$ from the center of the implant. Two magnifications of micrographs were used: $\times 270$ and $\times 2700$.

\section{Statistical analysis}

Statistical analyses were carried out using a specific software (GraphPad, CA, USA). The sample size was obtained assuming the standard deviation of 0.024 of the implant surface decontamination as the primary outcome [29]. It was calculated that 6 implants would be required in each group to have a fixed power of $80 \%$ with an alpha risk of $5 \%$ for the main variable. Data distribution was checked for normality by Shapiro-Wilk test. Kruskal-Wallis followed by Tukey's multiple comparisons test was used to compare the differences among groups. Data were presented as means \pm standard deviations (SDs) at each time (pre- and post-treatment) for every experimental group. Statistically significant differences were set to $p<0.05$.

\section{Results}

\section{Biofilm quantification}

The amount of biofilm present on the implants in the different groups was measured through CV staining and then expressed by means \pm standard deviation values of the $\mathrm{OD}_{595} \mathrm{~nm}$, which reflects the CV's absorbance (Table 1).

The human complex biofilm growth was successful as indicated by the statistical significance between the negative and positive controls. All the treatment groups (AA, $\mathrm{CAP}$, and $\mathrm{COM}$ ) resulted in a remarkable implant surface decontamination; statistically significant differences in the comparison between positive control vs. all the treatment groups were found. Besides, the groups AA and COM showed values of residual biofilm very close to the negative control with no statistical significance found in the comparison between negative control vs. AA $(p=0.1951)$ and vs. COM $(p=0.3013)$ as well as for AA vs. COM $(p=0.9930)$ (Table 2).

The measured percentage of biofilm removal revealed an effective decontamination following all the treatments and almost complete biofilm removal after both AA application (94.87\%) and when CAP was used in combination with AA in the COM treatment (95.32\%). CAP alone showed $52.10 \%$ of biofilm removal (Fig. 5).

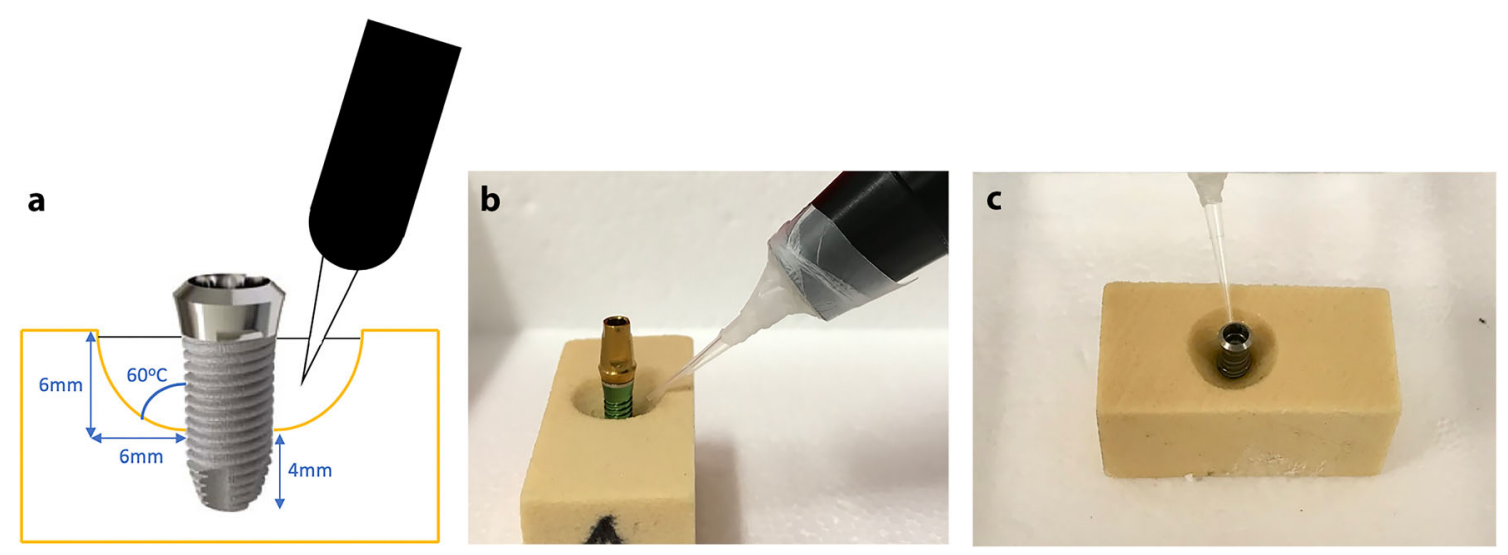

Fig. 4 a Schematic drawing of CAP treatment. b Setup of CAP treatment (NB: implant not representative). c Setup of CAP treatment 
Table 1 The amount of residual biofilm following decontamination treatments, measured through crystal violet staining and expressed by means \pm standard deviation values of the optical density measurements.

\begin{tabular}{lllll}
\hline -ve control & +ve control & AA & CAP & COM \\
\hline 0.0215 & $0.136 \pm 0.002$ & $0.030 \pm 0.003$ & $0.078 \pm 0.007$ & $0.029 \pm 0.004$ \\
\hline
\end{tabular}

Data are presented as mean \pm standard deviation (SD)

-ve control, non contaminated and treated by air abrasion and cold atmospheric plasma; +ve control, contaminated and untreated; AA, air abrasive; CAP, cold atmospheric plasma; COM, air abrasive + cold atmospheric plasma

Table 2 Results of Tukey's multiple comparison test for biofilm quantification

\begin{tabular}{lll}
\hline & Mean rank diff. & $p$ value \\
\hline -ve control vs. +ve control & -0.1145 & $* * * *<0.001$ \\
-ve control vs. AA & -0.008500 & 0.1951 \\
-ve control vs. CAP & -0.05650 & $* * *<0.001$ \\
-ve control vs. COM & -0.007500 & 0.3013 \\
+ve control vs. AA & 0.1060 & $* * * * 0.001$ \\
+ve control vs. CAP & 0.05800 & $* * * * 0.001$ \\
+ve control vs. COM & 0.1070 & $* * * *<0.001$ \\
AA vs. CAP & -0.04800 & $* * * * 0.001$ \\
AA vs. COM & 0.001000 & 0.9930 \\
CAP vs. COM & 0.04900 & $* * *<0.001$ \\
\hline
\end{tabular}

-ve control, non contaminated and treated by air abrasion and cold atmospheric plasma; +ve control, contaminated and untreated; AA, air abrasive; CAP, cold atmospheric plasma; COM, air abrasive + cold atmospheric plasma

$p$ values were based on Tukey's multiple comparison test

\section{Surface topography}

SEM analysis at different magnifications outlined no posttreatment alterations on the implants, such as crater-like defects or scratches, indicating that none of the applied treatments led to surface feature changes. In addition, the post-treatment SEM images revealed the significant removal of biofilm with the AA and COM treatments. In the +ve control at both low- and high-power magnification, presumable remnants of biofilm obscured the original implant surface (Fig. 6a-1).

\section{Discussion}

The ideal treatment for peri-implantitis should aim at the removal of the whole biofilm, the achievement of a sterile implant surface, and the maintenance of the implant surface topography; this in turn enhances host cell adhesion and re-osseointegration. The search for an efficient and safe approach for Ti surface decontamination has resulted in an effective treatment modality which is the AA treatment. Although there is no robust clinical evidence suggesting which decontamination modality is the most effective for treating peri-implantitis, a recent review [30] concluded that AA has a clinical efficacy which is superior or equal to all other decontamination methods (i.e.: Er:YAG laser, metal instruments and ultrasonic devices, plastic curettes, and rubber cups). Regarding the delivered
Fig. 5 Percentage of biofilm removal after the decontamination of Ti implants with a moderately rough surface by means of air-abrasive unit (AA) delivering erythritol, cold atmospheric plasma (CAP), and combined AA and CAP (COM) treatments

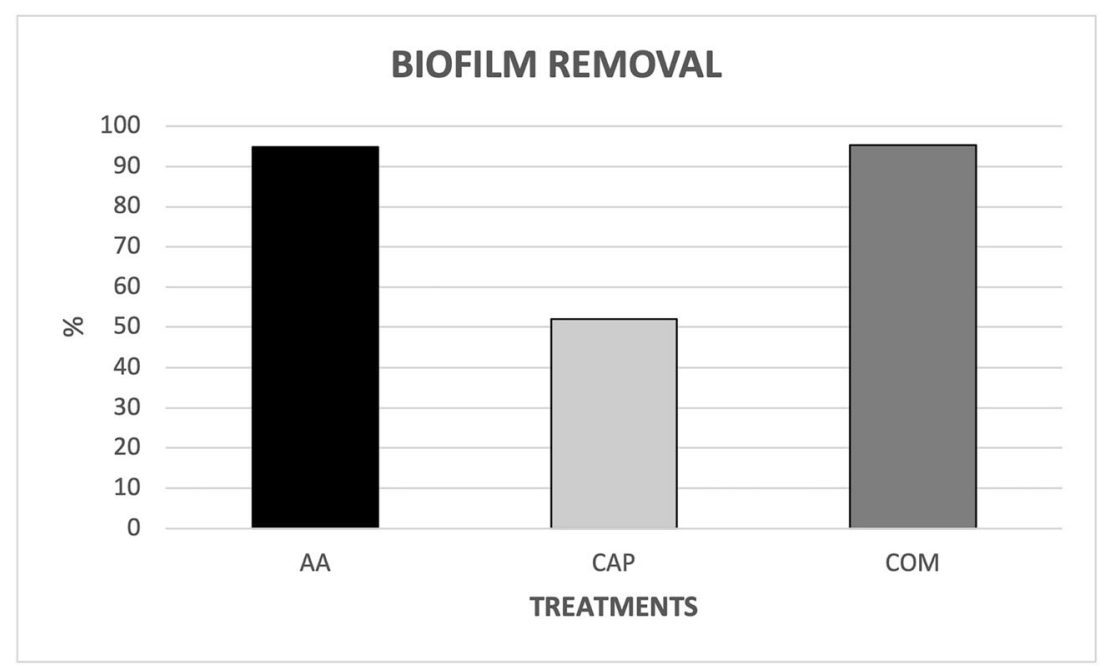



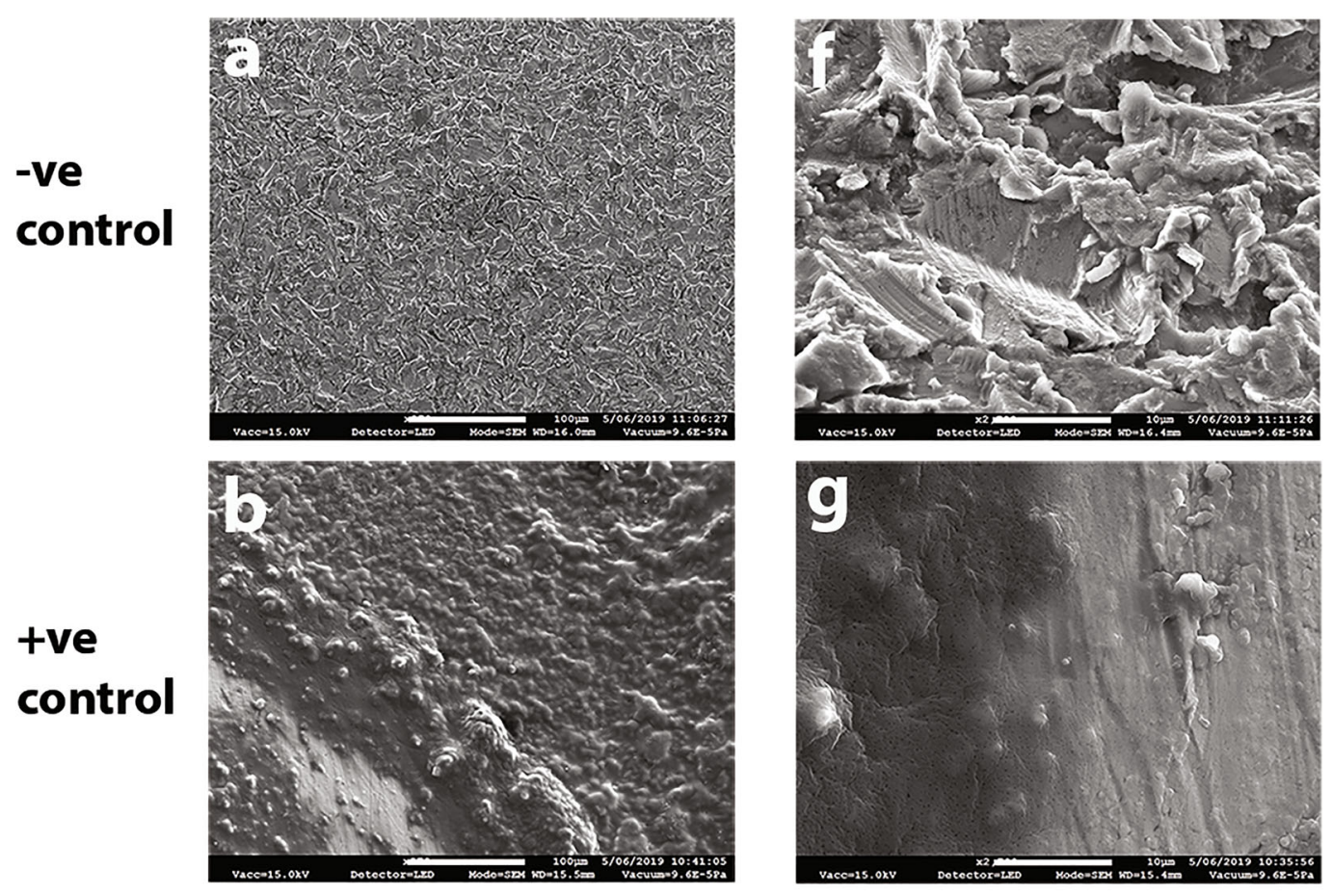

AA
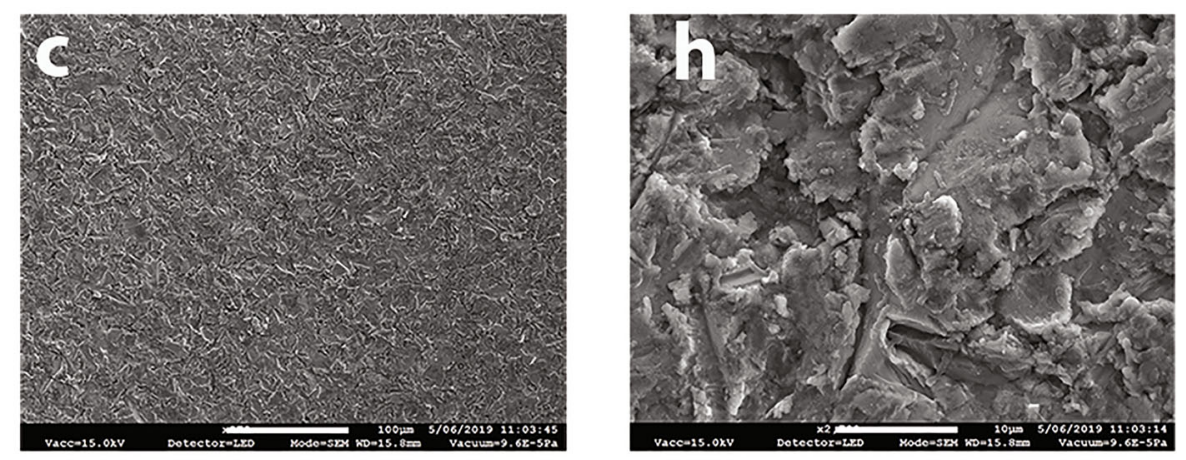

CAP
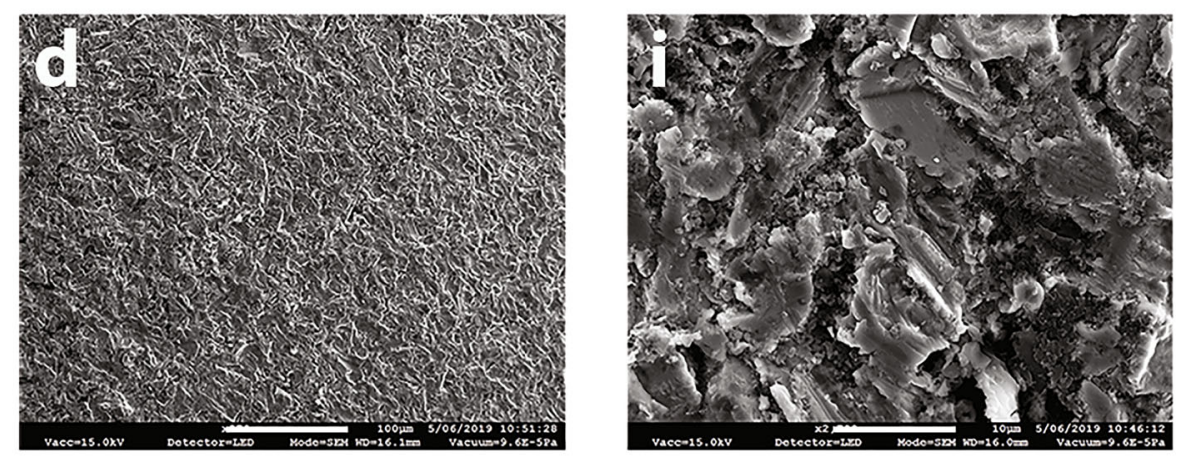

\section{COM}
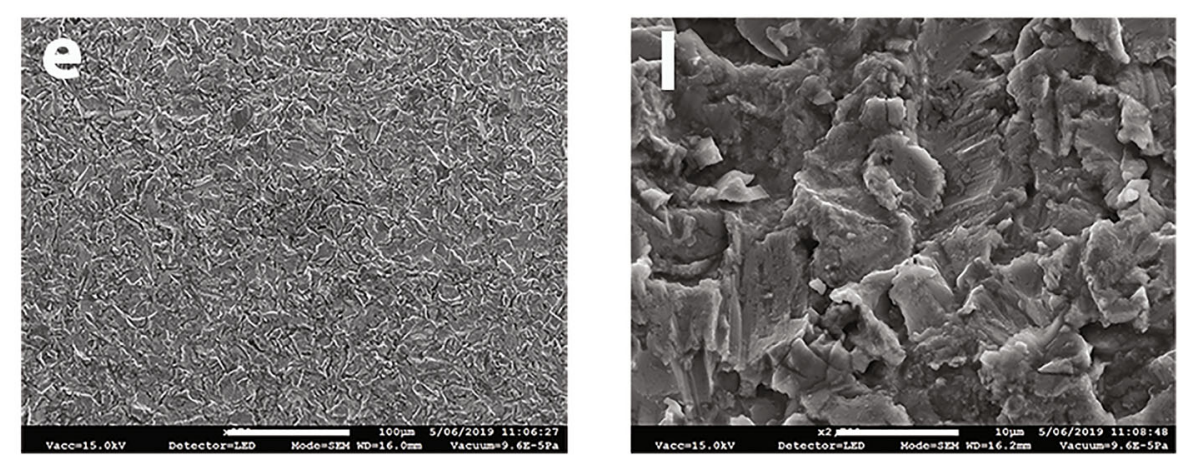
Fig. 6 Scanning electron microscope (SEM) images of the titanium implants after the decontamination with air-abrasive unit (AA), cold atmospheric plasma (CAP), combined AA and CAP (COM) treatments; negative (-ve) control, non-contaminated and treated by AA and CAP; positive (+ve) control group, contaminated and untreated discs. Scale bars are $100(\mathbf{a}-\mathbf{e})$ and $10(\mathbf{f}-\mathbf{I})$ micrometers, magnification: $\times 270(\mathbf{a}-\mathbf{e})$ and $\times 2700(\mathbf{f}-\mathbf{l})$.

powder, the best results were obtained using erythritol/ chlorhexidine in terms of preventing regrowth of oral biofilm [30]. Based on the evidence mentioned above in the present study, it was decided to investigate AA delivering erythritol and complement the treatment with CAP. Indeed, CAP should be seen not only as an alternative but also as an adjunct to investigate synergistic treatment modalities. Very few studies have been published in the literature on the effects of a combined treatment of CAP with other devices. In a recent review [18], only 17 in vitro studies and 5 in vivo on animal models investigating the effects of CAP on biocompatibility, surface improvement, and cleaning efficacy were found in the literature. Among these studies, only Matthes et al. [31] assessed the effects of a combined biofilm removal with an optimized AA with erythritol powder and an argon CAP on osteoblast-like cells spreading. They concluded that CAP alone did not render the surface conducive for cells and that the combination was not superior to AA alone. These results were confirmed by a very recent study by our group [27] where a relevant decontamination efficacy of both machined and moderately rough surfaces following AA and COM treatments was found. In detail, treatment with AA (99.92\% and 93.96) and COM (95.94\% and $88.55 \%)$ resulted in higher decontamination compared to CAP $80.9 \%$ and $42.63 \%$; however, no specific effect of the CAP in the combined treatment was observed. These results are in line with the ones achieved in the present study where AA showed $94.87 \%$ of biofilm removal, the combined treatment only a slight additional effect $(95.32 \%)$, and CAP alone $52.10 \%$, although it was used in a liquid environment. Plasma-liquid interactions have gained tremendous attention in the last few years; the transfer of reactivity from the gas to the liquid phase has been highlighted as being of prime importance for biological effects. Indeed, the depth of penetration of CAP can be limited to $60 \mu \mathrm{m}$, while CAP-activated liquid medium can act longer and deeper [32]. However, there are no indications in the literature about the exact "therapeutic dose"; the plasma gaseous products-and therefore the species generated in liquids - strongly depend on the discharge regime, its deposited power, and gas flow conditions. The gaseous products then determine the chemical properties of the CAP-activated liquid medium and the dominant aqueous reactive oxygen and nitrogen species (RONS). It was out of the scope of the present article to quantify the RONS produced by the investigated CAP device in our experimental conditions. Hence, an excessive dilution might be the cause of the slight effect of CAP alone and in combination.

The limitations of the present study are related to the restricted thickness of biofilms to several micrometers and the variation of the thickness due to its inhomogeneous growth. Another limitation concerns the plasma device itself. Indeed, it is an experimental device that was constructed to test different plasma parameters in vitro and to understand the principles of cold plasma generation. Therefore, further process optimization should be implemented to use it as a medical device, and its biocompatibility to oral mucosa should be investigated [33, 34]. Our results should be just understood mostly as a proof of principle. Nevertheless, we can state that plasma is an effective antimicrobial agent and further engineering, and optimization of the plasma sources is a viable way towards clinical translation.

\section{Conclusions}

Decontamination with AA delivering erythritol with or without CAP is highly effective in biofilm removal from titanium surfaces in our peri-implantitis model. All the tested treatments resulted in minimal titanium surface alterations that do not compromise the original surface features. Further studies are needed to confirm the decontamination effect of CAP as single treatment modality and its eventual additive or synergic effect when CAP is used in liquid medium combined with AA.

Acknowledgements The authors would like to kindly thank EMS and Southern Implants (Australia) for the kind and unconditioned support through the donation of part of the consumables and the Ti implants.

Author contribution All the authors above approved the final version of the manuscript. Specifically:

Wang Lai Hui contributed to conception and design, data collection, and analysis and drafted the manuscript. Vittoria Perrotti contributed to statistics, data analysis, and interpretation; drafted; and critically revised the manuscript. Adriano Piattelli contributed to data interpretation and critically revised the manuscript. Kostya (Ken) Ostrikov contributed to conception and critically revised the manuscript. Zhi Fang contributed to conception and critically revised the manuscript. Alessandro Quaranta contributed to conception and design, to data acquisition, and interpretation and critically revised the manuscript.

Funding Open access funding provided by Università degli Studi G. D'Annunzio Chieti Pescara within the CRUI-CARE Agreement. The work was self-funded by the authors and their institutions.

\section{Declarations}

Ethics approval All procedures performed in studies involving human participants were in accordance with the ethical standards of the Griffith University Human Research Ethics Committee (GU Ref No: 2018/35) 
and with the 1964 Helsinki declaration and its later amendments or comparable ethical standards.

Informed consent For this type of study, formal consent is not required.

Conflict of Interest The authors declare no competing interest.

Open Access This article is licensed under a Creative Commons Attribution 4.0 International License, which permits use, sharing, adaptation, distribution and reproduction in any medium or format, as long as you give appropriate credit to the original author(s) and the source, provide a link to the Creative Commons licence, and indicate if changes were made. The images or other third party material in this article are included in the article's Creative Commons licence, unless indicated otherwise in a credit line to the material. If material is not included in the article's Creative Commons licence and your intended use is not permitted by statutory regulation or exceeds the permitted use, you will need to obtain permission directly from the copyright holder. To view a copy of this licence, visit http://creativecommons.org/licenses/by/4.0/.

\section{References}

1. Derks J, Tomasi C (2015) Peri-implant health and disease. A systematic review of current epidemiology. J Clin Periodontol 42(Suppl 16):S158-S171. https://doi.org/10.1111/jcpe.12334

2. Berglundh T, Armitage G, Araujo MG, Avila-Ortiz G, Blanco J, Camargo PM, Chen S, Cochran D, Derks J, Figuero E, Hämmerle CHF, Heitz-Mayfield LJA, Huynh-Ba G, Iacono V, Koo KT, Lambert F, McCauley L, Quirynen M, Renvert S, Salvi GE, Schwarz F, Tarnow D, Tomasi C, Wang HL, Zitzmann N (2018) Peri-implant diseases and conditions: consensus report of workgroup 4 of the 2017 World Workshop on the Classification of Periodontal and Peri-Implant Diseases and Conditions. J Periodontol 89(Suppl 1):S313-s318. https://doi.org/10.1002/jper. 17-0739

3. Roccuzzo M, Layton DM, Roccuzzo A, Heitz-Mayfield LJ (2018) Clinical outcomes of peri-implantitis treatment and supportive care: a systematic review. Clin Oral Implants Res 29(Suppl 16):331-350. https://doi.org/10.1111/clr.13287

4. Albrektsson T, Wennerberg A (2004) Oral implant surfaces: part 1review focusing on topographic and chemical properties of different surfaces and in vivo responses to them. Int J Prosthodont 17(5): $536-543$

5. Albrektsson T, Wennerberg A (2004) Oral implant surfaces: part 2review focusing on clinical knowledge of different surfaces. Int $\mathrm{J}$ Prosthodont 17(5):544-564

6. Esposito M, Grusovin MG, Worthington HV (2012) Interventions for replacing missing teeth: treatment of peri-implantitis. Cochrane Database Syst Rev 1(1):Cd004970. https://doi.org/10.1002/ 14651858.CD004970.pub5

7. de Almeida JM, Matheus HR, Rodrigues Gusman DJ, Faleiros PL, Januário de Araújo N, Noronha Novaes VC (2017) Effectiveness of mechanical debridement combined with adjunctive therapies for nonsurgical treatment of periimplantitis: a systematic review. Implant Dent 26(1):137-144. https://doi.org/10.1097/id. 0000000000000469

8. Pesce P, Menini M, Tealdo T, Bevilacqua M, Pera F, Pera P (2014) Peri-implantitis: a systematic review of recently published papers. Int J Prosthodont 27(1):15-25. https://doi.org/10.11607/ijp.3785

9. Schwarz F, Schmucker A, Becker J (2015) Efficacy of alternative or adjunctive measures to conventional treatment of peri-implant mucositis and peri-implantitis: a systematic review and meta- analysis. Int J Implant Dent 1(1):22. https://doi.org/10.1186/ s40729-015-0023-1

10. Sahrmann P, Ronay V, Hofer D, Attin T, Jung RE, Schmidlin PR (2015) In vitro cleaning potential of three different implant debridement methods. Clin Oral Implants Res 26(3):314-319. https://doi. org $/ 10.1111 /$ clr. 12322

11. Schwarz F, Sahm N, Schwarz K, Becker J (2010) Impact of defect configuration on the clinical outcome following surgical regenerative therapy of peri-implantitis. J Clin Periodontol 37(5):449-455. https://doi.org/10.1111/j.1600-051X.2010.01540.x

12. Renvert S, Polyzois I, Claffey N (2012) Surgical therapy for the control of peri-implantitis. Clin Oral Implants Res 23(Suppl 6):84 94. https://doi.org/10.1111/j.1600-0501.2012.02554.x

13. Renvert S, Roos-Jansåker AM, Claffey N (2008) Non-surgical treatment of peri-implant mucositis and peri-implantitis: a literature review. J Clin Periodontol 35(8 Suppl):305-315. https://doi.org/10. 1111/j.1600-051X.2008.01276.x

14. Wei MCT, Tran C, Meredith N, Walsh LJ (2017) Effectiveness of implant surface debridement using particle beams at differing air pressures. Clinical and Experimental Dental Research 3(4):148153. https://doi.org/10.1002/cre2.74

15. Stock K, Hibst R (2008) Smart fiber tips for dental laser applications. Medical Laser Application 23(1):6-13. https://doi.org/10. 1016/j.mla.2007.11.002

16. Quaranta A, Maida C, Scrascia A, Campus G, Quaranta M (2009) Er:Yag Laser application on titanium implant surfaces contaminated by Porphyromonas gingivalis: an histomorphometric evaluation. Minerva Stomatol 58(7-8):317-330

17. Otsuki M, Wada M, Yamaguchi M, Kawabata S, Maeda Y, Ikebe K (2020) Evaluation of decontamination methods of oral biofilms formed on screw-shaped, rough and machined surface implants: an ex vivo study. Int J Implant Dent 6(1):18-18. https://doi.org/ 10.1186/s40729-020-00212-y

18. Hui WL, Perrotti V, Iaculli F, Piattelli A, Quaranta A (2020) The emerging role of cold atmospheric plasma in implantology: a review of the literature. Nanomaterials (Basel) 10(8). https://doi.org/ 10.3390/nano10081505

19. Schwarz F, Sager M, Ferrari D, Herten M, Wieland M, Becker J (2008) Bone regeneration in dehiscence-type defects at nonsubmerged and submerged chemically modified (SLActive) and conventional SLA titanium implants: an immunohistochemical study in dogs. J Clin Periodontol 35(1):64-75. https://doi.org/10. 1111/j.1600-051X.2007.01159.x

20. Wang XQ, Wang FP, Chen W, Huang J, Bazaka K, Ostrikov KK (2016) Non-equilibrium plasma prevention of Schistosoma japonicum transmission. Sci Rep 6:35353. https://doi.org/10.1038/ srep35353

21. Tresp H, Hammer MU, Winter J, Weltmann KD, Reuter S (2013) Quantitative detection of plasma-generated radicals in liquids by electron paramagnetic resonance spectroscopy. Journal of Physics D: Applied Physics 46(43):435401. https://doi.org/10.1088/00223727/46/43/435401

22. Liu F, Sun P, Bai N, Tian Y, Zhou H, Wei S, Zhou Y, Zhang J, Zhu W, Becker K, Fang J (2010) Inactivation of bacteria in an aqueous environment by a direct-current, cold-atmospheric-pressure air plasma microjet. Plasma Processes and Polymers 7(3-4):231-236. https://oi.org/10.1002/ppap.200900070

23. Sánchez MC, Llama-Palacios A, Blanc V, León R, Herrera D, Sanz M (2011) Structure, viability and bacterial kinetics of an in vitro biofilm model using six bacteria from the subgingival microbiota. J Periodontal Res 46(2):252-260. https://doi.org/10.1111/j.16000765.2010.01341.x

24. Estrela C, Sydney GB, Figueiredo JA, Estrela CR (2009) A model system to study antimicrobial strategies in endodontic biofilms. J Appl Oral Sci 17(2):87-91. https://doi.org/10.1590/s167877572009000200003 
25. Koop HM, Valentijn-Benz M, Nieuw Amerongen AV, Roukema PA, De Graaff J (1989) Aggregation of 27 oral bacteria by human whole saliva. Influence of culture medium, calcium, and bacterial cell concentration, and interference by autoaggregation. Antonie Van Leeuwenhoek 55(3):277-290. https://doi.org/10.1007/ bf00393856

26. Schwarz F, Herten M, Sager M, Bieling K, Sculean A, Becker J (2007) Comparison of naturally occurring and ligature-induced peri-implantitis bone defects in humans and dogs. Clin Oral Implants Res 18(2):161-170. https://doi.org/10.1111/j.1600-0501.2006. 01320.x

27. Hui WL, Ipe D, Perrotti V, Piattelli A, Fang Z, Ostrikov K, Quaranta A (2021) Novel technique using cold atmospheric plasma coupled with air-polishing for the treatment of titanium discs grown with biofilm: an in-vitro study. Dent Mater 37(2):359-369. https:// doi.org/10.1016/j.dental.2020.11.027

28. Wang G, Feng H, Hu L, Jin W, Hao Q, Gao A, Peng X, Li W, Wong KY, Wang H, Li Z, Chu PK (2018) An antibacterial platform based on capacitive carbon-doped $\mathrm{TiO}(2)$ nanotubes after direct or alternating current charging. Nat Commun 9(1):2055. https://doi. org/10.1038/s41467-018-04317-2

29. Guarnieri R, Rappelli G, Piemontese M, Procaccini M, Quaranta A (2016) A double-blind randomized trial comparing implants with laser-microtextured and machined collar surfaces: microbiologic and clinical results. Int J Oral Maxillofac Implants 31(5):11171125. https://doi.org/10.11607/jomi.4563
30. Moharrami M, Perrotti V, Iaculli F, Love RM, Quaranta A (2019) Effects of air abrasive decontamination on titanium surfaces: a systematic review of in vitro studies. Clin Implant Dent Relat Res 21(2):398-421. https://doi.org/10.1111/cid.12747

31. Matthes R, Duske K, Kebede TG, Pink C, Schluter R, von Woedtke T, Weltmann KD, Kocher T, Jablonowski L (2017) Osteoblast growth, after cleaning of biofilm-covered titanium discs with airpolishing and cold plasma. J Clin Periodontol 44(6):672-680. https://doi.org/10.1111/jcpe. 12720

32. Khlyustova A, Labay C, Machala Z, Ginebra M-P, Canal C (2019) Important parameters in plasma jets for the production of RONS in liquids for plasma medicine: a brief review. Frontiers of Chemical Science and Engineering 13(2):238-252. https://doi.org/10.1007/ s11705-019-1801-8

33. Jablonowski L, Kocher T, Schindler A, Muller K, Dombrowski F, von Woedtke T, Arnold T, Lehmann A, Rupf S, Evert M, Evert K (2019) Side effects by oral application of atmospheric pressure plasma on the mucosa in mice. PLoS One 14(4):e0215099. https://doi.org/10.1371/journal.pone.0215099

34. Liu D, Xiong Z, Du T, Zhou X, Cao Y, Lu X (2011) Bacterialkilling effect of atmospheric pressure non-equilibrium plasma jet and oral mucosa response. J Huazhong Univ Sci Technolog Med Sci 31(6):852-856. https://doi.org/10.1007/s11596-011-0690-y

Publisher's note Springer Nature remains neutral with regard to jurisdictional claims in published maps and institutional affiliations. 